\title{
Brief Review of Clinical Morphological and Therapeutic Profiles of the Hand and Wrist Osteoarticular Tuberculosis
}

\section{Dan Nelu Anușca ${ }^{1,2,3 *}$ and Raul Filip Mureșan ${ }^{2}$}

${ }^{1}$ Doctoral School, University of Medicine and Pharmacy of Craiova, Romania

${ }^{2}$ Department of Orthopaedics and Traumatology, Emergency County Hospital of

Craiova, Romania

${ }^{3}$ Department of Orthopaedics and Traumatology, University of Medicine and

Pharmacy of Craiova, Romania

*Corresponding Author: Dan Nelu Anușca, Doctoral School, University of Medicine and Pharmacy of Craiova, Romania.
Received: May 26, 2021

Published: July 19, 2021

(C) All rights are reserved by Dan Nelu Anușca and Raul Filip Mureșan.

\section{Abstract}

Tuberculosis (TB) is an endemic disease known since the Paleolithic in animal species, even before it affected humans. Today it presents serious concerns for global health, being the second most infectious disease after malaria, causing the most deaths worldwide despite the remarkable progress made in recent decades in terms of screening, monitoring and therapeutic strategy. In 2017 there were 1.8 million deaths worldwide from tuberculosis, but we are witnessing a very topical reappearance [1,2]. There are several factors that contribute today to maintaining this status of global tuberculosis health problem with its recurrence in developed countries. These are: immigration from regions where the disease is endemic; increasing number of elderly people with debilitating diseases; increasing the number of immuno-compromised patients; the appearance of mycobacterium-multidrug-resistant strains; increasing drug, alcohol, unemployment, poverty and malnutrition abuse; the HIV epidemic, tuberculosis being often the first manifestation of HIV infection; Extrapulmonary tuberculosis (TBEP) is more common in Asian and African countries, having today a fantastic increase from $7.6 \%$ in 1970 to $35-40 \%$ [3-5].

Osteoarticular tuberculosis (OATB) increased in incidence in 35\% of extrapulmonary cases, especially in underdeveloped countries [6-8].

Two basic types of disease patterns could be present: the granular type (most often in adults) and the Cassius exudative type (most often in children), one of which being predominant. The algorithm of diagnosis includes several steps of which detection of Mycobacterium tuberculosis (Mt) is the gold standard. The actual treatment is primarily medical, consisting of antituberculosis chemotherapy (ATT), surgical intervention being warranted only for selected cases. It is essential that clinicians know and refresh their knowledge about the manifestation of OATB [9].

Keywords: Osteoarticular Tuberculosis; Bone and Joint Tuberculosis; Extrapulmonary Tuberculosis; Hand and Wrist

\section{Introduction}

OATB was identified 9,000 years ago at the spine level in Egyptian mummies, and is today a rare form of TB [5,10-12]. Wrist and hand tuberculosis is becoming a fairly common form of the disease today if we consider the difficulty of reporting and underdiagnosis today. Risk factors or predisposers of OATB are: increasing number of people with suppression of the immune system in chronic immuno-suppressive diseases, immuno-suppressive therapy and patients with HIV and AIDS: 
- $\quad$ The development of resistant and multi-resistant Mycobacterium tuberculosis strains;

- Increased exposure of health professionals to patients positive for TB;

- Multiparous or lactation women, color population, drug abuse and alcohol;

- Increasing the number of patients with diabetes mellitus, chronic kidney failure, chronic obstructive bronchopneumopathy (BPOC), hepatic cirrhosis, lympho-proliferative diseases;

- The older population is increasing;

- The decline in the public health network's interest for controlling TB [5];

- Immigration from countries with high tuberculosis prevalence (Africa, Asia);

- $\quad$ Socio-economic factors such as poverty, homeless people, protein malnutrition, poor hygiene, housing congestion [5].

The OATB pathogenesis of the hand and wrist is most often hematogenous, but also lymphatic, as well as by direct invasion or by lesion contiguity.

The diagnosis is made epidemiologically (great attention to the origin and passage through different countries of citizens and their contacts with patients), clinical, radiological, CT scan and MRI.

Clinically tuberculosis of the hand and wrist causes local symptoms with pain, limited and difficult movements, joint stiffness, lymphadenopathy, cold abscesses and fistulization.

Paraclinic tuberculin skin test (TST), enzyme linked immunosorbent assay (ELISA), interferon gamma release assay (IGRA).

Plane radiography denotes soft tissue edema, osteopenia, osteoporosis, osteolysis with a characteristic radiological image "in the mirror", subchondral erosion, narrowing of the subchondral space, cysts, appearance of "spina ventosa".

MRI accurately delineates the involvement of soft tissues and lesions associated with adjacent bones and joints [13].

CT scan helps detect the involvement of bone and joint destruction. Tomography has a special role in guiding percutaneous biopsy and drainage of abscesses [5].
Detection of Mt by:

- Ziehl-Neelsen and immunohistochemistry stains on smears and histological slides.

- $\quad$ Culture of Mt from bone/synovial/soft tissue/draining sinuses, synovial fluid although, in some cases, cultures may reveal colonizing bacteria or fungi that are erroneously assumed to be causative pathogen.

- $\quad$ Polymerase chain reaction (PCR) $[5,9]$.
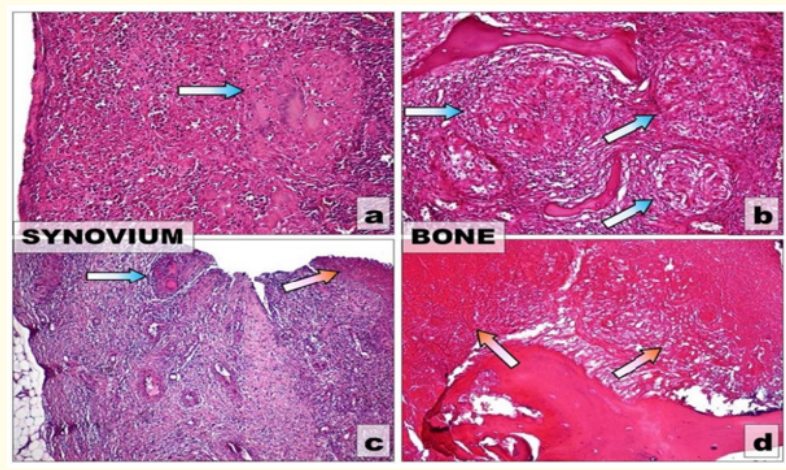

Figure 1: Main histological patterns of OATB. UP: Granular typeGiant Langhans cell granulomas (blue arrows) Placed (a) in the synovium, (b) in the cancellous bone, H-E stain, x10; DOWN:

Caseous necrosis (red arrows) (a) in the synovium $x 4$ (b) in the compact bone, x10, H-E stain.

The material used for Mt detection could be:

- Synovial fluid or purulent infected material obtained by aspiration or fine needle aspiration biopsy;

- Bone/synovial/soft tissue obtained by biopsy.

The traditional criteria for diagnosing TB are:

- $\quad$ Chest radiology.

Detection of acid-fast bacilli by Ziehl-Neelsen stain on microscopy and culture. Microscopy is the most rapid diagnostic tool, but it is very insensitive, yielding only $10-30 \%$ of culture-positive samples [9,14-16].

Biopsy may be required to clear up diagnostic confusion, being the most definitive test for tuberculous arthritis. It must be per- 
formed in cases in which microbiology test gives negative results, the demonstration of caseating granulomas on histological examination being of significant value. Culture is the gold standard for the diagnosis of osseous TB in the culture of Mycobacteria from bone tissue or synovial fluid, but may take four weeks to obtain conclusive results even with enhanced culture systems.

Newer methods of diagnosis, especially the polymerase chain reaction (PCR) on obtaining joint tissue biopsies, appears promising in the early diagnosis of tuberculous arthritis.

Drug susceptibility testing of isolates is essential. In the respect, the Xpert MTB/RIF assay is an automated nucleic acid amplification test that can simultaneously identify Mt and rifampin resistance; it has been shown to be fast and accurate in diagnosing musculoskeletal TB in children and adults [5,17].

\section{Differential diagnosis}

The differential diagnosis of skeletal TB includes:

- Subacute or chronic infection due to pathogens or diseases such as (depending upon epidemiologic factors):

- $\quad$ Staphylococcus aureus osteomyelitis;

- Brucellosis;

- Melioidosis;

- Actinomycosis;

- $\quad$ Candidiasis;

- Histoplasmosis;

- Metastatic malignancy (especially multifocal bone involvement).

- Malignancy (metastases, multiple myeloma, lymphoma) $[5,9]$.

Treatment

There is medical, surgical and auxiliary treatment.

\section{Medical treatment}

Under strictly survayed treatment (SST) associated with C, D and $B$ vitamin group over a period of twelve months. If during the last three months the results are positive the 3 drugs based therapy can be introduced in SST, also associated with C, D and B vitamin group, remaining on 3 drugs for another minimum three months:
Hydrazide - 300 mg/day (5 - 10 mg/day), Rifampicin - 600 mg/day (10 mg/kg/day), Pyrazinamide - 1,5 g/day (25 - $30 \mathrm{mg} / \mathrm{kg} /$ day), Ethambutol - $1 \mathrm{~g} /$ day (15 mg/kg/day) or Streptomycin - $1 \mathrm{~g} /$ day, if the results treatment based on clinical, radiography and CT scan investigations allow it.

\section{Surgical treatment}

Involves surgical release by rehabilitation in the carpal tunnel, surgical arthrodesis of the fist with or without metal fixation, associated with local treatment with tuberculostatic drug filling or bone, after lesion cleansing and fixation in a functional position $[9,18]$.

\section{Auxiliary treatment}

Plaster immobilization to prevent deformities of infected extremities $[19,20]$. In our opinion, the interphalangeal joint prosthesis becomes insufficient with all the inactivity of the disease at a distance of at least 10 years due to its lack of effectiveness. Surgical indication is required in the absence of drug treatment results after 4 months of treatment [21,22].

\section{Conclusion}

The incidence of the tuberculosis process evolves differently depending on the location of the tuberculosis in the skeleton. The articular forms such as OTB of the hand and the wrist have a temporal evolution with an oscillating tendency with an ascending general trend in the middle age range and of the young adult, especially in the male sex.

The suspicion of the etiological diagnosis at the clinical examination is generally high when TB was not suspected, the suspicion was directed towards arthritic diseases with immunological determinism in the hand, or neoplasms.

The granulomatous reaction is of poorly differentiated or even disorganized type with incipient hyperactive lesions, without necrosis.

We insist on the study of particular morphological profiles depending on the affected tissue, so the morphological picture can provide indications of the aggressiveness of Mycobacterium tuberculosis, the quality of the host's immune response or the temporal evolution of lesions, such as their predilection to extension $[5,9]$. 


\section{Acknowledgements}

Illustration of histological and imaging aspects belong to OATB cases hospitalized in Pediatric Surgery and Orthopaedics and Traumatology departments of the Emergency County Hospital of Craiova, Romania [5,9].

\section{Bibliography}

1. "Global Tuberculosis Report". World Health Organization 3 (2017).

2. Z Yang., et al. "Identification of risk factors for extrapulmonary tuberculosis". Clinical Infectious Diseases 38.2 (2004): 199205.

3. I Grosskopf., et al. "Bone and joint tuberculosis: a 10 year review”. Israel Medical Association Journal 30.4 (1994): 278-283.

4. S Gunal., et al. "Demographic and microbial characteristics of extrapulmonary tuberculosis cases diagnosed in Malatya, Turkey, 2001-2007". BMC Public Health 11 (2011): 154-161.

5. I Procopie and D N Anusca. Teza de doctorat- Contrinuții la studiul profilului clinico-morfologic al tuberculozei osteoarticulare, Ș. D. U. Craiova, Ed., Craiova: Universitatea de Medicină și Farmacie Craiova (2017): 130.

6. A Malaviya and P Kotwal. "Arthritis associated with tuberculosis". Best Practice and Research: Clinical Rheumatology 17.2 (2003): 319-343.

7. C Pigrau-Serrallach and D Rodriguez-Pardo. "Bone and Joint tuberculosis”. European Spine Journal 22.4 (2013): 556-566.

8. A Houston and D Macallan. "Extrapulmonary tuberculosis". Medicine 42.1 (2014): 18-22.

9. I Procopie., et al. "Diagnosis and treatment particularities in Osteoarticular Tuberculosis". Current Health Sciences Journal 43.2 (2017).

10. H Donoghue. "Human tuberculosis is an ancient disease, as elucidated by acient microbial biomolecules". Microbes Infection 11.14-15 (2009): 1156-1162.

11. R Garg and D Somvanshi. "Spinal tuberculosis: a review". Journal of Spinal Cord Medicine 34.5 (2011): 440-454.

12. H Watt and R Lifeso. "Current Concepts Review: Tuberculosis of Bones and Joints". The Journal of Bone and Joint Surgery 78.2 (1996): 288-298.
13. A Thrush and D Enzmann. "MR imaging of infectious spondylitis". American Journal of Neuroradiology 11.6 (1990): 11711180.

14. G Versfeld and A Solmon. "A diagnostic approach to tuberculosis of bones and joints". The Journal of Bone and Joint Surgery 64.4 (1982): 446-449.

15. A Mondal. "Cytological diagnosis of vertebral tuberculosis whit fine-needle aspiration biopsy". The Journal of Bone and Joint Surgery 76.2 (1994): 181-184.

16. P Merino., et al. "Microbiological diagnosis of spinal tuberculosis". International Orthopaedics 36.2 (2012): 233-238.

17. M Held., et al. "Diagnostic accuracy of tge Xpert MTB/RIF Assay for extrapulmonary tyberculosis in children with musculoskeletal infections". Pediatric Infectious Disease Journal 35.11 (2016): 1165-1168.

18. M Subasi., et al. "Tuberculosis of the metacarpals and phalanges of the hand". Annals of Plastic Surgery 53.5 (2004): 469472.

19. M Al-Qattan., et al. "Tuberculosis of the hand". Journal of Hand Surgery (American Volume) 36.8 (2011): 1413-1421.

20. S Chen., et al. "Long-term retrospecive analysis of surgical treatment for irretrievable tuberculosis of the ankle". Foot and Ankle International 34.3 (2013): 372-337.

21. S Chen and K Chen. "Updated Diagnosis and Management of Osteoarticular Tuberculosis". Journal of Emergency Medicine Trauma and Surgical Care 1 (2014): 1-5.

22. W Sequeira., et al. "Osteoarticular tuuberculosis: current diagnosis and treatment". American Journal of Therapy 7.6 (2000): 393-398.

\section{Volume 5 Issue 8 August 2021}

(C) All rights are reserved by Dan Nelu Anușca and Raul Filip Mureșan.

Citation: Dan Nelu Anușca and Raul Filip Mureșan. "Brief Review of Clinical Morphological and Therapeutic Profiles of the Hand and Wrist Osteoarticular Tuberculosis". Acta Scientific Medical Sciences 5.8 (2021): 61-64. 